\title{
International symposium of junior neuroscience investigators (ISJNI19) UPM, 25-27 April, 2019 (Part -II)
}

Parkinson's disease and therapeutic outcomes: From classic medication to therapy revolutionary

Ahmed Draoui ${ }^{1,2}$, Omar EL Hiba ${ }^{1,2}$, Abdelaati EL Khiat ${ }^{1}$, Halima Gamrani $^{1}$

${ }^{1}$ Neuroscience, Pharmacology and Environment Unit, faculty of Sciences Semlalia, Cadi Ayyad University, Avenue My Abdellah, B.P. 2390, Marrakesh, Morocco

${ }^{2}$ Nutrition and Food Sciences laboratory, faculty of Sciences, ChouaibDoukkali University El Jadida, Route Ben Maachou, B.P. 20, Avenue des Facultés, El Jadida, Morocco

Parkinson's disease (PD) is the most common neurodegenerative disease worldwide after Alzheimer's disease, with a prevalence of $0.3 \%$ in the world population. The cardinal symptoms of PD are mainly akinesia, bradykinesia, rigidity and resting tremor. Carlson's work in 1959 showed that parkinsonian motor symptoms are related to a decrease in dopamine (DA) $(-60 \%$ to $-80 \%)$ in the striatum following progressive and irreversible degeneration of dopaminergic neurons within the substantianigra pars compacta $(\mathrm{SNc})(-40 \%$ to $-50 \%)$. It is well established that the loss of DA within this pathway leads to a deregulation of a major network of neurons involved in the control of the development of movement, namely the basal ganglia. Current treatments for the disease focus on symptomatic management by restoring dopaminergic activity: drug treatments with L-Dopa, DA precursor, or by dopaminergic agonists, or by high frequency deep neurostimulation of the sub-thalamic nucleus (STN) without restoring neurodegeneration.

The current hope is cell therapy. Initial efforts in this area have relied on the use of fetal mesencephalon tissue as a source of DA neurons for transplantation, and then use of other cellular sources, such as stem cells or reprogrammed cells.

Recently, in vivo direct reprogramming of adult glia cells has emerged as a potentially revolutionary approach to cell replacement therapy that would prevent cell transplantation. Studies have been able to develop a new strategy in which dopaminergic neurons are generated by the direct conversion of human astrocytes in vitro and mouse astrocytes in vivo by uses of transcription factors and microRNAs.

This therapeutic strategy has given very promising results, especially for striatal astrocytes of adult mice reprogrammed to become functional DA neurons, leading to the restoration of locomotors deficits.

Key Words: parkinson's disease, dopamine, neurodegeneration, motor disorders, treatments, cell therapy, transplantation, reprogramming (in vivo / in vitro), astrocyte, dopamine neuron

\section{Neurobehavioral disorders observed after scorpion venom administration}

Moulay Abdelmonaim EL Hidan, Mehdi AIT Laaradia, Abdelmohcine Aimrane, Ahmed Draoui, Mohamed Echchakery and Kholoud Kahime

Faculty of Applied Sciences, Ait Melloul, Ibn Zohr University

Scorpion toxins act on ion channels (sodium, potassium, calcium and chlorine).The effects of these toxins on ion channels could promote an impairment that may result in an abnormal release of neurotransmitters, known to be involved in the control of several cognitive behaviors. In the present investigation, we assessed the possible neurobehavioral disturbances occurring in mice envenomed with $\mathrm{H}$. gentili venom. After the subcutaneous injection of $0.4 \mathrm{mg} / \mathrm{kg}$ of $\mathrm{H}$. gentili crude venom the behavioral observations were made $30 \mathrm{~min}, 3 \mathrm{~h}$ and $12 \mathrm{~h}$ post envenomation. Our results show that the neurobehavioral impairments are time dependent. In fact, administration of $\mathrm{H}$. gentili crude venom decreased locomotion and rearing frequencies and increased the time of immobility and grooming of the animals tested $30 \mathrm{~min}$ and $3 \mathrm{~h}$ post injection. Besides the locomotor's changes, this work clearly demonstrated the time dependent anxiolytic effects of H. gentili venom in the lightedark choice test. Venom also had induced a depression state especially $30 \mathrm{~min}$ and $3 \mathrm{~h}$ post injections. Different fractions of the venom toxins are necessary for the complete understanding of the present results.

Key Words: scorpion venom, neurobehavioral impairments, locomotor activity, depression, anxiety

\section{Altered working memory and hippocampal astroglia morphology in rat with thioacetamide-induced acute hepatic encephalopathy}

Abdelaati EL Khiat*, Omar EL Hiba, Lahcen Tamegart, Ahmed Draoui, Radouane EL Fari, Souad Sellami, Hanane Rais and Halima Gamrani

Neurosciences, Pharmacology and Environnement Unit (NPEU), faculty of sciences Semlalia, Cadi Ayyad University, Marrakesh, Morocco

Hepatic encephalopathy (HE) is a complex neuropsychiatric syndrome resulting from acute or chronic hepatic impairments. The

${ }^{\star}$ Correspondence to: Omar EL Hiba, Department of Biology, Chouaib Doukkali University, Route Ben Maachou, 24000, El Jaddia, Morocco, E-mail: omar. elhiba@edu.uca.ac.ma

Received: April 19, 2019; Accepted: April 26, 2019; Published: April 30, 2019 
clinical features of HE includes attention as well as a mild cognitive deficit associated with impaired attentional and executive networks in patients as well as in animal models of HE. The underlining pathomechanism of memory impairment in HE patients is still not fully understood, however, it may involve a possible gliopathy as well as neuropathy. The aim of the present investigation is to assess progression of short working memory deterioration in acute HE and to delineate the glial and the neuronal alteration which may underlie such cognitive impairment. The study was carried out in male Sprague-Dawley rats with acute liver failure induced by thioacetamide (TAA). The study was performed on different stages of acute HE, 12h, 24h and 36h following administration of TAA. The liver functions were assessed via different biochemical markers (ALT, AST, bilirubin, urea and creatinine) and a histopathological examination of the liver tissue. While for the behavioral study, we used T-Maze test to assess short working memory using the percentage of alternation behavior, together with an immunohistochemical analysis of the Glial Fibrillary Acidic Protein (GFAP) as the key marker of astrocytes in the hippocampus, as well as serotonin (5-HT) for 5-HTergic neurons within the dorsal Raphe nucleus (DRN). Our data revealed a progressive loss of liver tissue integrity with inflammation and hepatocytes degeneration, which was associated to obvious loss of the liver function. In parallel, we observed a gradual alteration of the alternation behavior, as a sign of altered short working memory in the acute HE rats. At the central level, the immunohistochemical study showed a time dependent region-specific changes of GFAP-immunoreactive astrocytes within the hippocampus. While within the DRN, serotonin levels declined progressively in a timedependant manner. Our data revealed for the first time, a gradual loss of short memory function in acute HE, resulting from liver dysfunction. Such cognitive deterioration may involve a possible gliopathy as well as a 5-HTergic dysfunction which could be considered as a new key element for understanding the basis of memory and attention loss in HE patients.

Key words: acute hepatic encephalopathy, T-maze, short working memory, GFAP, serotonin, rat, dorsal raphe nucleus, hippocampus

\section{Impairement of the nigrostriatal dopaminergic innervation in rat with chemically-induced acute liver failure: A possible cause of parkinsonism in hepatic encephalopathy}

Abdelaati EL Khiat*, Mohamed Aitihya, Omar EL Hiba, Lahcen Tamegart and Halima Gamrani

Neurosciences, Pharmacology and Environnement Unit (NPEU), faculty of sciences Semlalia, Cadi Ayyad University, Marrakesh, Morocco

Hepatic encephalopathy (HE) is a progressive neuropsychiatric disorder that manifests itself in both acute and chronic liver failure. The presence of extrapyramidal symptoms, including rigidity and tremors, may reflect alterations of several neurotransmitters, including dopamine, GABA, glutamate or serotonin. The present study aimed to evaluate dopaminergic, serotonergic, and possible correlations with locomotor activity in an animal model of acute hepatic encephalopathy in rats. Induction of acute hepatic insufficiency was achieved in rats by repeated i.p administrations of thioacetamide. Using immunohistochemistry and the open-field assay, we evaluated respectively tyrosine hydroxylase (TH) immunoreactivity in substantia nigra pars compacta (SNpc) and locomotor activity. Our results showed a significant decrease in the level of TH immunoreactivity in SNpc concomitant with hypoactivity in TAA rats compared to controls. the present study seems to sustain the loss of DA innervations within the brain structures known to be directtly involved in motor function control, therefore, our finding may be considered as on of the underlying pathomechanisms of parkinsonism in hepatic encephalopathy.

Key words: acute HE, dopamine, substantia nigra pars compacta, parkinsonism, rat, locomotor activity

\section{Protective effect of Omega-3 in manganese-induced locomotor impairments}

Radouane EL Fari ${ }^{*}$, Abdellatif Abbaoui, Abdeljalil EL Got and Halima Gamrani

Neurosciences, Pharmacology and Environment Unit, faculty of sciences Semlalia, Cadi Ayyad University, Marrakesh, Morocco

Manganism is a syndrome presenting cognitive and motor deficits, with shared features with Parkinson's disease. Many cases were reported since the mid of 19th century, in welding, metallurgic and miners, in Morocco too, nowadays, sources of exposure to manganese $(\mathrm{Mn})$ are expanding to comprise pesticides, dry batteries industry, and several other ways. Despite its essentiality overexposure to $\mathrm{Mn}$ induces motor impairments, hence, prevention is highly required. Recent studies have shown Omega-3(n-3) to have a nutraceutical potential declining neurodegeneration, therefore, aiming to assess the protective effect of some Omega-3 PUFAs against Mn-induced locomotor impairments, we used a sub-acute Mn animal model, and we assessed spontaneous activity, coordination, and strength in animals treated by $\mathrm{Mn}, \mathrm{Mn}$ and n-3, n-3 alone and control. Results showed a significant recovery of locomotor ability in all its forms of DHA in co-treated animals compared to the intoxicated group, while no differences were noticed in n-3 alone group. Based on this finding PUFAs should certainly make part of a normal diet given their protective potential proved in the present study and many other on neurodegenerative diseases like Alzheimer and Parkinson's diseases

Key words: manganese, manganism, locomotor behavior, omega-3, movement disorder, parkinson's disease

\section{Interaction of Mn with MPTP and 6OHDA in Parkinson's disease animal models}

Radouane EL Fari*, Abdellatif Abbaoui, Abdeljalil EL Got and Halima Gamrani

Neurosciences, Pharmacology and Environment Unit, faculty of sciences Semlalia, Cadi Ayyad University, Marrakesh, Morocco

Manganese is a metallic trace element the 5th most abundant in the earth crust, given its wide use in different industries, exposition to immoderate amounts of Mn cause Manganism an extrapyramidal syndrome with parkinsonian-like symptoms. It was thought that Mn would be only a factor exacerbating parkinsonian features in Parkinson's disease famous animal models (MPTP and 6OHDA). Nevertheless, it's not the case, indeed co-treatment with Mn and MPTP showed a decrease in extrapyramidal impairments when compared to MPTP group, on the other hand, co-treatment with Mn and 6OHDA showed Mn to emphasis 6OHDA effects exacerbating Parkinsonian features such as locomotor behavior, in the present review we show the mechanisms underlying this interaction between Mn/MPTP and $\mathrm{Mn} / 6 \mathrm{OHDA}$, in order to reveal Mn proprieties floating from protective to powerful neurotoxicant. 
Key words: manganese, locomotor behavior, movement disorder, parkinson's disease, pd animal models

\section{Copper neurotoxicity involvement in neurodegenerative diseases: Current insights}

\section{Abdellatif Abbaoui*}

Faculte des sciences semlalia, Université Cadi Ayad, Marrakesh, Marocco

Copper is an essential trace element for human body, since it is a cofactor of several enzymes and proteins and plays a key role in numerous important biological processes (e.g., respiration, protection from oxidative damage, iron metabolism, etc.), also including the central nervous system development and functioning (e.g. synthesis of neurotransmitters, myelination, activation of neuropeptides, etc.). Then, copper homeostasis is tightly regulated, but dysregulation of copper (deficiency or copper overload) homeostasis is associated with different neurobehavioral injuries and neurotoxic effects, mainly represented by oxidative stress, and it has been described in several neurodegenerative disorders, such as Parkinson's disease, Wilson's disease, Menkes disease, Alzheimer's disease, and Amyotrophic Lateral Sclerosis. Herein, we will describe potential mechanisms of the cellular toxicity of copper, the current knowledge on the connection of copper toxicity with pathophysiology of neurodegenerative diseases. Additionally, a hint on novel therapeutic approaches based on restoring copper homeostasis through metal chelators will be pointed out.

Key words: copper, neurotoxicity, oxidative stress, neurodegenerative diseases, natural compounds

\section{Serotonin involvement in the subcommissural organ-Reissner's fiber complex response to acute and subchronic copper exposure in rats and modulatory effect of curcumin}

Abdellatif Abbaoui ${ }^{\star}$, Lahcen Tamegart, Radouane EL Fari and Halima Gamrani

Cadi Ayyad University, Faculty of sciences Semlalia, Neurosciences, Pharmacology and Environment Unit, Marrakesh, Morocco

Metal neurotoxicity is a universal health preoccupation. Previous data revealed an obvious neurochemical impairment induced by metal elements as copper. This investigation was conducted to study the subcommissural organ (SCO) response to acute and subchronic $\mathrm{Cu}$ exposure as well as its serotoninergic innervation in Wistar rats, and the probable protective potential of curcumin in these toxicological circumstances. By mean of immunohistochemistry using antibodies against Reissner's fiber (RF) and serotonin (5HT) in acute model (10 $\mathrm{mg} / \mathrm{kg}$ i.p. for 3 days) and subchronic model $(0.125 \%$ in drinking water for six weeks), we noted a significant decrease of RF-immunoreactivity and a whole amplified 5-HT innervation of SCO and ventricular borders in intoxicated rats. Co-treatment with curcumin-I $(30 \mathrm{mg} /$ $\mathrm{kg} \mathrm{B.W)} \mathrm{has} \mathrm{shown} \mathrm{a} \mathrm{beneficial} \mathrm{effect,} \mathrm{reinstating} \mathrm{both} \mathrm{SCO} \mathrm{secretory}$ activity and serotoninergic innervation damaged by $\mathrm{Cu}$ exposure. This data revealed for the first time an obvious response of SCO-RF complex to $\mathrm{Cu}$ intoxication as well as the neuroprotective effect of curcumin-I. Thus, SCO could play a fundamental role in the strategies of brain resistance to neurotoxicity induced by metal elements in rats, and may be used as biomarker to assist in the diagnosis of this neurotoxicological conditions in rodents.

Key words: copper, subcommissural organ, reissner's fiber, serotonin, curcumin, rats

Copyright: $\odot 2019$ Hiba OEL. This is an open-access article distributed under the terms of the Creative Commons Attribution License, which permits unrestricted use, distribution, and reproduction in any medium, provided the original author and source are credited. 